\title{
UV-induced DNA self-repair mechanism traced with quantum chemistry
}

\author{
Sebastian Reiter ${ }^{1}$, Daniel Keefer ${ }^{1}$, Vitus Besel $^{1}$, and Regina de Vivie-Riedle ${ }^{1,{ }^{*}}$ \\ ${ }^{1}$ Department Chemie, Ludwig-Maximilians-Universität München, Butenandtstr. 11, 81377 München, \\ Germany
}

\begin{abstract}
Recently, UV radiation was found to promote the self-repair of a damaged DNA nucleobase sequence. The proposed mechanism after photoexcitation of an adjacent guanine adenine sequence is now validated by our quantum chemical calculations.
\end{abstract}

\section{UV excitation can lead to self-repair of a DNA lesion}

Nucleobases as the fundamental building blocks of DNA and RNA are susceptible to UV radiation. The UV photons can trigger photochemical and photophysical processes and potentially lead to DNA photodamage [1]. Mostly, the high photostability of the nucleobases is attributed to ultrafast relaxation back to the electronic ground state [2]. Nevertheless, DNA lesions occur frequently and need to be repaired by the organism. A recent experimental study discovered that UV radiation can not only damage genetic material but also promote self-repair of defective nucleobase sequences [3]. The authors performed illumination experiments on double stranded oligonucleotides containing the cyclobutane pyrimidine dimer $(\mathrm{CPD})$ lesion adjacent to a guanine $(\mathrm{G})$ adenine $(\mathrm{A})$ sequence (oligonucleotide: G-A-CPD-A-G, cf. fig. 1). They found that excitation of G leads to repair of the CPD lesion and claim a long-living (300 ps) charge-transfer state between $\mathrm{G}$ and $\mathrm{A}$, followed by electron transfer from A to the CPD to be responsible for the repair. With the tools of state of the art quantum chemistry (QC), we investigate this mechanism by inspecting the possibilities of exciplex formation and the character and energetic profile of the excited states that can be reached with the experimentally applied UV excitation at $290 \mathrm{~nm}$.

\footnotetext{
* Corresponding author: Sebastian.Reiter@cup.uni-muenchen.de
} 

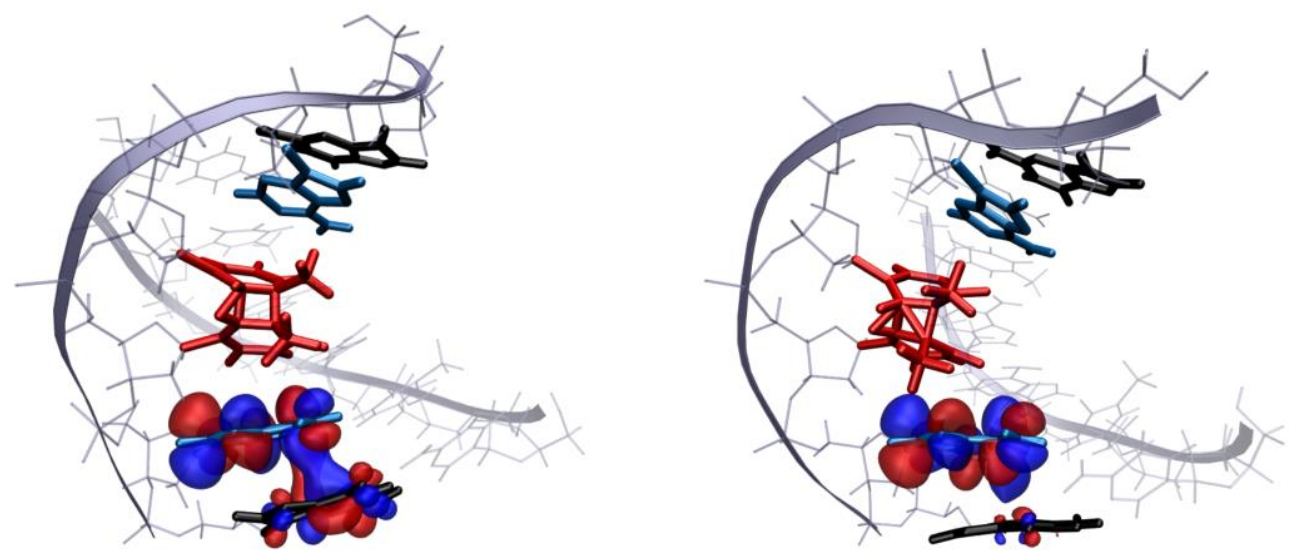

Fig. 1. Oligonucleotide containing the CPD lesion (two covalently bound thymines, red), framed by two adenine (light blue) guanine (black) sequences and with the complementary strand. This structure was used experimentally [3], as well as for our theoretical calculations. In the left geometry, there is an orbital overlap between guanine and adenine indicating exciplex formation, while the right geometry shows no overlap of any orbitals.

\section{Exciplex formation after photoexcitation}

The first step of the proposed photochemically initiated self-repair of the CPD lesion involves the formation of G-A exciplexic states. To examine this exciplex formation, we took isolated $\mathrm{G}$ and $\mathrm{A}$ and positioned them with their molecular planes parallel to each other, as it is the case in the oligonucleotide structure. We then scanned several orientational coordinates, like the G-A distance (z-direction), the $\mathrm{x}$ - and y-translation of the two nucleobases (cf. fig. 2) and their rotation within the molecular plane. This orientational scan includes geometries commonly occurring in a molecular dynamics (MD) simulation which we performed for the complete oligonucleotide (fig. 1). The orbitals of many G-A orientations were computed with time-dependent density functional theory (TDDFT), and for some geometries there is significant orbital overlap (left part in fig. 1), while for other geometries there is no overlap (right part in fig. 1). We then computed the lowest five electronically excited states of all G-A geometries and checked for excitations which have major contributions into exciplexic orbitals. For quantification purposes, we introduced a parameter $X_{E}$ for the exciplexic strength of the geometry, which is calculated as follows:

$$
X_{E}=\frac{1}{N} \sum_{n=1}^{N} \frac{\sum_{e} c_{e}}{\sum_{e} c_{e}+\sum_{a} c_{a}}
$$

Here, $N$ is the number of excited states considered (usually the lowest five), $c_{e}$ are excitation coefficients with contribution into overlapping (exciplexic) orbitals and $c_{a}$ are excitation coefficients without contribution from overlapping orbitals. $X_{E}$ can take values from 0 (no exciplex character) to 1 (strong exciplex character) and is depicted with a color code for an exemplary orientational scan in fig. 2 . 


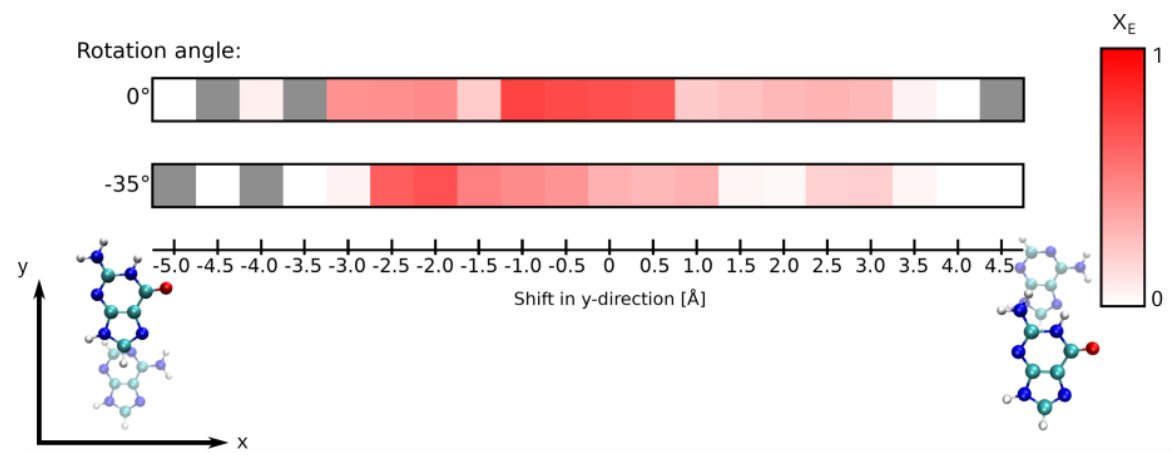

Fig. 2. Exciplexic strength along an exemplary orientational scan between guanine and adenine. The parameter $X_{E}$ is calculated according to eq. (1). It can take values between 1 (strong exciplexic character) and 0 (no exciplexic character) and is encoded in red color according to the bar on the right. The direction of the scan is indicated on the bottom left, with a guanine rotation within its molecular plane of $0^{\circ}$ (according to the depicted geometries) and $35^{\circ} . X_{E}$ takes the largest values at overlapping geometries. The oscillatory behavior (maximum values at $-2.5 \AA, 0 \AA$ and $2.5 \AA$ in the $0^{\circ}$ scan) is due to one ring, two rings and again one ring overlapping.

In this example, $\mathrm{G}$ and $\mathrm{A}$ are shifted in y-direction, with a $\mathrm{G}$ rotation in the molecular plane of $0^{\circ}$ and $35^{\circ}$. As expected, $X_{E}$ takes large values where $\mathrm{G}$ and A show the most overlap. However, in both examples there is a periodic feature, which corresponds to geometries where only part of the molecules are on top of each other. The latter case is often found in the MD trajectory of the oligonucleotide, and thus we conclude that exciplexic states are existent and no rare events in the DNA sequence. This means that the first step of the experimentally proposed self-repair mechanism can be confirmed.

\section{Involved excited states characterized with CASPT2 QM/MM}

To accurately describe the energetic position and electronic character of the excited states, more sophisticated QC methods are needed. We therefore took few exemplary snapshots of the oligonucleotide MD trajectory and used the multiscale quantum mechanics / molecular mechanics (QM/MM) approach, where the GA sequence is treated with high-level QM while the rest of the strand is treated classically. For the QM region, we used state of the art CASPT2 with active space sizes containing up to 12 electrons in 12 active orbitals. In contrast to the isolated GA system, the excitation energies within the DNA strand are lowered to the experimentally addressed region. We found excited states solely located at $\mathrm{G}$ together with close lying excited states where electron density is shifted from $G$ to A. This suggests that the proposed second step of the UV induced self-repair - namely a long-living GA charge transfer state - is conceivable and within the possibilities of the calculated excited states in the oligonucleotide.

\section{References}

1. M. Barbatti, A. C. Borin, S. Ullrich, eds., Photoinduced Phenomena in Nucleic Acids (Springer International Publishing, Switzerland, 2015)

2. C. E. Crespo-Hernandez, B. Cohen, P. M. Hare, B. Kohler, Chem. Rev. 104, 1977 $2020(2004)$

3. D. B. Bucher, C. L. Kufner, A. Schlueter, T. Carell, W. Zinth, J. Am. Chem. Soc. 138, $186-190(2016)$ 